\title{
Properties of Synchronous Versus Metachronous Bilateral Breast Carcinoma with Long Time Follow Up
}

\author{
Nuket Eliyatkin ${ }^{1 *}$, Baha Zengel ${ }^{2}$, Ayse Yagci $^{3}$, Erdem Comut ${ }^{3}$, Hakan Postaci $^{3}$, \\ Adam Uslu' ${ }^{2}$, Safiye Aktas ${ }^{4}$
}

\begin{abstract}
Background: Breast cancer is the most common cancer type among women with increasing incidence rates, improved prognosis and survival. According to the localization of the tumor, breast cancer is designated as unilateral (UBC) or bilateral (BBC). BBC can be classified as synchronous (SBBC) or metachronous (MBBC) based on the time interval between the diagnosis of the first and the secondary tumors. According to the guideline of WHO 2012, BBC is generally defined as SBBC when contralateral breast carcinoma is diagnosed within 3 months. The aim of this study was to compare the characteristics and patterns of metastasis of BBC patients with UBC. Materials and Methods: A cohort of 768 patients with breast cancer treated at the Turkish Ministry of Health-Izmir Bozyaka Research and Training Hospital between 1976 and 2012 were studied. Survival analysis was performed comparing UBC and BBC patients. In addition, evaluations were performed in patients with SBBC and MBBC sub-groups. We used a 3-months interval to distinguish metachronous from synchronous. Results: When clinical and histopathological parameters were statistically evaluated, ER status, event-free and overall survival were found to be significant between UBC and BBC patients. In comparison of SBBC and MBBC patients, age, histological type of tumor, event-free and overall survival were found to be significant. Conclusions: BBC cases were found to show worse prognosis than UBC cases. Among BBC, SBBC had the worst prognosis based on overall survival rates.
\end{abstract}

Keywords: Bilateral breast cancer - unilateral breast cancer - metachronous - synchronous

Asian Pac J Cancer Prev, 16 (12), 4921-4926

\section{Introduction}

Breast cancer is the most common cancer type among women which has increasing incedence rates, improved prognosis and survival (Hartman 2007). Various breast cancer types may present with different clinical and biological characteristics According to the localization of the tumor, breast cancer is designated as unilateral and bilateral. The unilateral breast cancer (UBC) is defined when the tumor is appeared in only one breast tissue. If the tumor arise in each breast tissue, this type of breast carcinoma is called bilateral breast cancer (BBC). Among all breast cancers BBC accounts 2-6\% and the development risk of $\mathrm{BBC}$ is two to six fold higher in women who already had primary UBC (Hartman 2007, Chen 1999).

BBC can be classified as synchronous and metachronous based on the time interval between the diagnosis of the first and the secondary tumors. Some previous studies have defined synchronous bilateral breast cancer (SBBC) as the presentation of breast cancer within 0-12 months of each other (Chen 2013). According to the guideline of WHO 2012, BBC is generally defined as SBBC when contralateral breast carcinoma is diagnosed within 3 months. If contralateral breast carcinoma is diagnosed more than 3 months, this type of $\mathrm{BBC}$ is defined as metachronous bilateral breast carcinoma (MBBC) (Hartman 2007, Irvine 2009). SBBC is also defined as the presentation of breast cancer within 12 months in studies from an epidemiological point of view (Huo 2011, Ibrahim 2015). The SBBC is found to be relatively low between 1 and $3 \%$ of all cases of breast cancers (Holm 2014). SBBC and MBBC shows distinct biological characteristics which are mainly reflecting differences in histopathological features, stage and prognosis (Senkus 2014).

Some studies showed that BBCs are associated with worse outcome and reduced survival. On the contrary, some other studies claimed that there is no significant different in survival between UBC and BBC. Despite the previous and recent ongoing studies, the impact of the type of $\mathrm{BBC}$ on the prognosis and the survival of the patients, still remains uncertain. (Yoshimura 2013).

${ }^{1}$ Pathology, Faculty of Medicine, Adnan Menderes University, Aydin, ${ }^{2}$ General Surgery, Turkish Ministry of Health-Izmir Bozyaka Research and Training Hospital, Izmir, ${ }^{3}$ Pathology, Turkish Ministry of Health-Izmir Bozyaka Research and Training Hospital, ${ }^{4}$ Basic Oncology Institute of Oncology, Dokuz Eylul University, Izmir, Turkey, *For correspondence: drnuket2003@yahoo.com 
The aim of this study to compare the characteristics and patterns of metastasis of BBC patients with UBC. Survival analysis was performed comparing UBC and $\mathrm{BBC}$ patients. In addition same evaluations were performed in patients with sub-groups of BBC as SBBC and $\mathrm{MBBC}$.

\section{Materials and Methods}

\section{Patients}

We identified 768 patients (760 female, 8 male) who were diagnosed as breast cancer and treated for an invasive primary breast cancer at Turkish Ministry of HealthIzmir Bozyaka Research and Training Hospital between 1976 and 2012. We collected clinical and pathological information of the patients from clinical archive files until October 2014. UBC patients were followed up for 1- 293 months $( \pm 81.65)$. BBC patients were followed up for 10 401 months $( \pm 117.2)$. Median follow up period was 90 months. 8 patients were excluded because no further data was achieved after diagnosis. The patients were operated for mastectomy or prophylactic mastectomy. We evaluated estrogen receptor (ER), progesteron receptor $(\mathrm{PgR})$ and CerB2 status, TNM stages, location and number of metastasis, subtype as unilateral or bilateral, SBBC and MBBC types and survivals. Mammographical image of a SBBC patient was shown in Figure 1. Our outcome of interest were local recurrence or distant metastasis. Distant metastases were defined as any lesion located outside the breast tissue, mastectomy scar, ipsilateral axillary lymph nodes or ipsilateral supraclavicular lymph nodes. Metastatic sites were grouped as: bone, lung, liver, central nervous system, gynecological, distant cutaneous; distant lymph nodes; multiple organs. We used a 3-months interval to distinguish metachronous from synchronous CBC.

\section{Statistical analysis}

Statistical analysis was performed by using SPSS 16.0 software (for Windows, Chicago, IL). Descriptives and frequencies of the parameters in the study were evaluated with standard derivation. The correlation analysis was
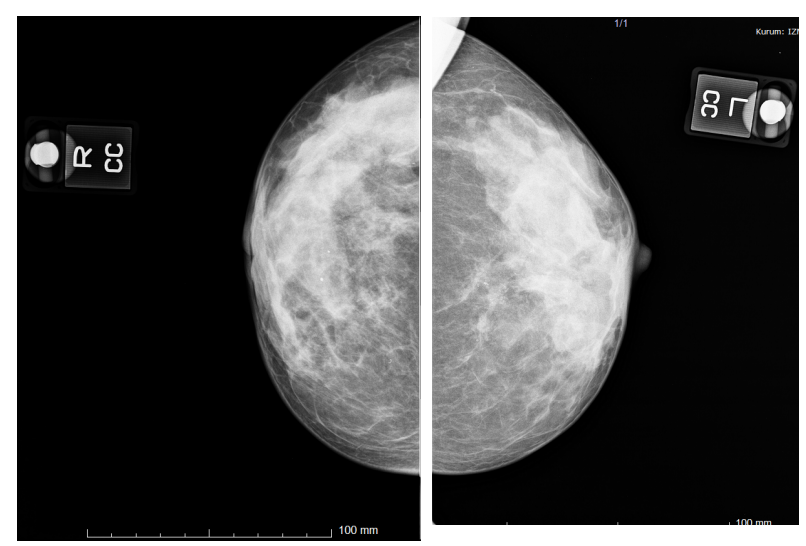

Figure 1. Senkronous Bilateral Breast Carcinoma Mammography done by Pearson correlation test. Statistical analysis were performed by Chi Square test, Mann Whitney U nonparametric test and Independent sample t test. Survival analysis was performed by Kaplan Meier test with log rank analysis. Pvalues $<0.05$ was considered to be statistically significant.

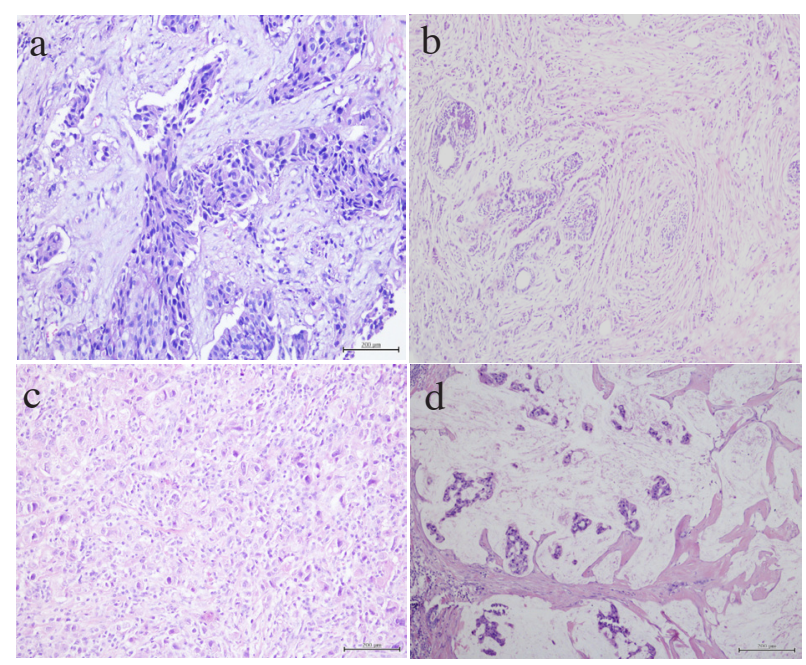

Figure 2. a) Invasive Ductal Carcinoma; b) Invasive Lobular Carcinoma; c) Carcinoma with Signet-Ring Cell; d) Mucinous Carcinoma
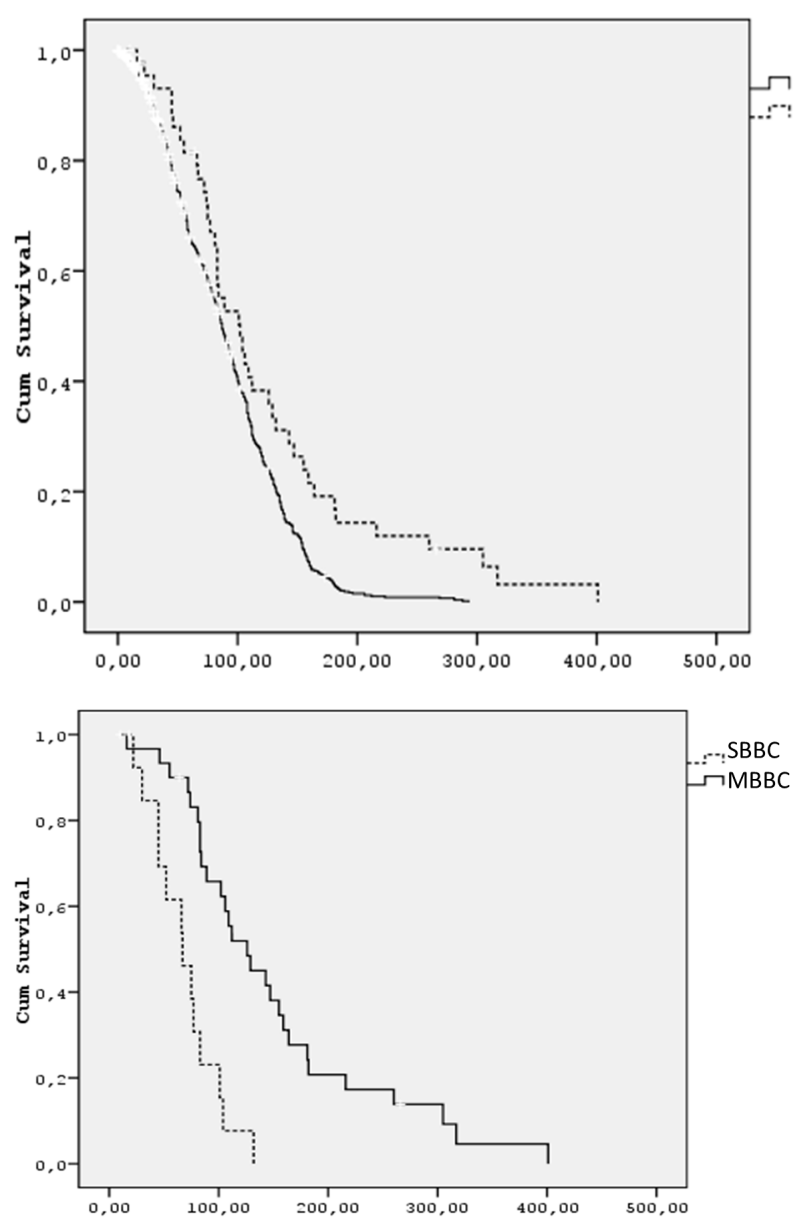

Figure 3. a) Overall Survival Analysis; b) Overall Survival Analysis 


\section{Results}

This study included 768 patients with breast cancer. 45 of them were BBC either metachronous $(n=30)$ or synchronous $(n=15)$. Eight of the cases were male (all of them were unilateral). $39.5 \%$ of the cases had used birth control pills in the past. $43.5 \%$ of the cases were premenopause. $85.6 \%$ of the cases had underwent modified radical mastectomy, $12.5 \%$ cases lumpektomy/ quadrantectomy. $65.8 \%$ of the cases were diagnosed as invasive ductal carcinoma, $10.8 \%$ of the cases were diagnosed as invasive lobular carcinoma and $23.6 \%$ of the cases were diagnosed as other types of breast cancer. $59.4 \%$ of the cases were ER positive, $59.1 \%$ of the cases were PgR positive. $66.4 \%$ of the cases were cerbB 2 score $0 / 1+, 12.5 \%$ of the cases were cerbB2 score 2 and $21.1 \%$ of the cases were cerbB2 score 3. p53 was positive in $20.1 \%$ of the cases. Ki67 proliferation index was high (over $14.0 \%$ ) in $26,3 \%$ of the cases. $15.7 \%$ of the cases were stage I, $40.9 \%$ of the cases were stage II and $33.2 \%$ of the cases were stage III. Stage was not accessed in $8.1 \%$ of the cases. $4.9 \%$ of the cases had local recurrence. $14.1 \%$ of the cases had bone metastasis while $10.2 \%$ of the cases had lung metastasis, $8.8 \%$ of the cases had liver metastasis and $2 \%$ of the cases had brain metastasis. $45.4 \%$ of the cases were right sided breast tumor and $48.7 \%$ of the cases were left sided breast tumor. 45 of the cases had BBC (5.9\%). 15 of the 45 BBC cases had synchronous tumor (33.3\%). 30 of the 45 bilateral breast cancer cases had metachronous breast cancer $(66.7 \%)$ ( $\mathrm{p}>0.005$ Chi Square test). The descriptives and frequencies of the clinical and pathological characteristics of all cases were as shown in Table 1.

When histopathologic diagnosis of the groups compared, the invasive ductal carcinoma whether in combination with ductal component or not, was observed in $65.7 \%$ of cases in unilateral group, besides it was $75.6 \%$ in bilateral group at the first breast diagnosis (Figure 2). $46.6 \%$ of the synchronous cases were in the same histopathologic morphology of both sides. Besides $60 \%$

Table 1. Comparison of clinicopathological characteristics (unilateral breast carcinoma versus first tumor of BBC).

\begin{tabular}{|c|c|c|c|}
\hline Characteristics & Unilateral $(\mathrm{n}=723)$ & First tumor of $\mathrm{BBC}(\mathrm{n}=45)$ & P value \\
\hline Median age (years) (range) & $52.79 \pm 12.8$ & $52.55 \pm 11.31$ & $\mathrm{p}=0.167$ \\
\hline \multicolumn{4}{|l|}{ Histological types: } \\
\hline Invasive ductal carcinoma & $62 \%$ & $66.7 \%$ & \multirow[t]{4}{*}{$\mathrm{p}=0.12$} \\
\hline Invasive lobular carcinoma & $9.7 \%$ & $12.8 \%$ & \\
\hline Combined carcinoma & $9.3 \%$ & $7.7 \%$ & \\
\hline Others & $18 \%$ & $12.8 \%$ & \\
\hline Tumor size (mean value) & $3.22 \pm 1.88 \mathrm{~cm}$ & $3.41 \pm 2.16 \mathrm{~cm}$ & $\mathrm{p}=0.574$ \\
\hline \multicolumn{4}{|l|}{ ER status: } \\
\hline Positive & $58.5 \%$ & $73.2 \%$ & \multirow[t]{2}{*}{$\mathrm{p}=0.043$} \\
\hline Negative & $41.5 \%$ & $26.8 \%$ & \\
\hline \multicolumn{4}{|l|}{ PgR status: } \\
\hline Positive & $58.5 \%$ & $69.2 \%$ & \multirow[t]{3}{*}{$\mathrm{p}=0.122$} \\
\hline Negative & $41.5 \%$ & $10.8 \%$ & \\
\hline \multicolumn{3}{|l|}{ cerbB2 status: } & \\
\hline Positive & $20.7 \%$ & $25 \%$ & \multirow[t]{3}{*}{$\mathrm{p}=0.078$} \\
\hline Negative & $67.3 \%$ & $55 \%$ & \\
\hline Equivocal & $12 \%$ & $20 \%$ & \\
\hline \multicolumn{4}{|l|}{ Ki67 proliferation index: } \\
\hline$<14 \%$ & $73.6 \%$ & $72.2 \%$ & \multirow[t]{2}{*}{$\mathrm{p}=0.898$} \\
\hline$\geq 14 \%$ & $26.4 \%$ & $27.8 \%$ & \\
\hline \multicolumn{4}{|l|}{ Nodal status: } \\
\hline No & $37.3 \%$ & $40.9 \%$ & \multirow{4}{*}{$\mathrm{p}=0.518$} \\
\hline N1 & $24.8 \%$ & $25 \%$ & \\
\hline $\mathrm{N} 2$ & $18.1 \%$ & $18.2 \%$ & \\
\hline N3 & $13.9 \%$ & $11.4 \%$ & \\
\hline \multicolumn{4}{|l|}{ TNM stage: } \\
\hline Stage I & $15.6 \%$ & $17.8 \%$ & \multirow{4}{*}{$\mathrm{p}=0.169$} \\
\hline Stage II & $39.5 \%$ & $48.9 \%$ & \\
\hline Stage III & $33.8 \%$ & $24.4 \%$ & \\
\hline Stage IV & $2.6 \%$ & $6.7 \%$ & \\
\hline \multicolumn{4}{|l|}{ Metastasis: } \\
\hline Positive & $24.7 \%$ & $28.9 \%$ & \multirow[t]{2}{*}{$\mathrm{p}=0.319$} \\
\hline Negative & $75.3 \%$ & $71.1 \%$ & \\
\hline \multicolumn{4}{|l|}{ Metastatic site: } \\
\hline Bone & $6.7 \%$ & $6.2 \%$ & \multirow{3}{*}{$\mathrm{p}=0.864$} \\
\hline Others (lung, liver, brain) & - & - & \\
\hline None & $75.4 \%$ & $73.3 \%$ & \\
\hline \multicolumn{4}{|l|}{ Survival: } \\
\hline Alive & $64.1 \%$ & $62.2 \%$ & \multirow[t]{2}{*}{$\mathrm{p}=0.0001$} \\
\hline Ex & $22.7 \%$ & $28.9 \%$ & \\
\hline
\end{tabular}


Table 2. Comparison of clinicopathological characteristics (synchronous BBC versus metachronous BBC)

\begin{tabular}{|c|c|c|c|}
\hline Characteristics & Synchronous BBC $(n=15)$ & Metachronous BBC $(n=30)$ & $\mathrm{P}$ value \\
\hline Mean age (years) (range) & $59.86 \pm 11.18$ & $49.13 \pm 9.78$ & $\mathrm{p}=0.005$ \\
\hline \multicolumn{4}{|l|}{ Histological types: } \\
\hline Invasive ductal carcinoma & $42.8 \%$ & $79.2 \%$ & \multirow[t]{4}{*}{$\mathrm{p}=0.032$} \\
\hline Invasive lobular carcinoma & $14.2 \%$ & $12.5 \%$ & \\
\hline Combined carcinoma & $21.4 \%$ & $8.3 \%$ & \\
\hline Others & $21.4 \%$ & - & \\
\hline Tumor size (mean value, $\mathrm{cm}$ ) & $3.49 \pm 1.53$ & $3.38 \pm 2.43$ & $\mathrm{p}=0.468$ \\
\hline \multicolumn{4}{|l|}{ ER status: } \\
\hline Positive & $76.9 \%$ & $71.4 \%$ & \multirow[t]{2}{*}{$\mathrm{p}=0.513$} \\
\hline Negative & $23.1 \%$ & $28.6 \%$ & \\
\hline \multicolumn{4}{|l|}{ PgR status: } \\
\hline Positive & $84.6 \%$ & $61.5 \%$ & \multirow[t]{2}{*}{$\mathrm{p}=0.134$} \\
\hline \multicolumn{3}{|l|}{ cerbB2 status: } & \\
\hline Positive & $15.4 \%$ & $29.6 \%$ & \multirow[t]{3}{*}{$\mathrm{p}=0.514$} \\
\hline Negative & $53.8 \%$ & $55.6 \%$ & \\
\hline Equivocal & $30.8 \%$ & $14.8 \%$ & \\
\hline \multicolumn{4}{|l|}{ Ki67 proliferation index: } \\
\hline$<14 \%$ & $91.7 \%$ & $62.5 \%$ & \multirow[t]{2}{*}{$\mathrm{p}=0.069$} \\
\hline$\geq 14 \%$ & $8.3 \%$ & $37.5 \%$ & \\
\hline \multicolumn{4}{|l|}{ Nodal status: } \\
\hline NO & $21.4 \%$ & $50 \%$ & \multirow[t]{4}{*}{$\mathrm{p}=0.771$} \\
\hline N1 & $42.9 \%$ & $16.7 \%$ & \\
\hline $\mathrm{N} 2$ & $28.6 \%$ & $13.3 \%$ & \\
\hline N3 & $7.1 \%$ & $13.3 \%$ & \\
\hline \multicolumn{4}{|l|}{ TNM stage: } \\
\hline Stage I & $13.3 \%$ & $20 \%$ & \multirow[t]{4}{*}{$\mathrm{p}=0.489$} \\
\hline Stage II & $46.7 \%$ & $50 \%$ & \\
\hline Stage III & $26.7 \%$ & $23.3 \%$ & \\
\hline Stage IV & $13.3 \%$ & $3.3 \%$ & \\
\hline \multicolumn{4}{|l|}{ Metastasis: } \\
\hline Positive & $20 \%$ & $33.3 \%$ & \multirow[t]{2}{*}{$\mathrm{p}=0.285$} \\
\hline Negative & $80 \%$ & $66.7 \%$ & \\
\hline \multicolumn{4}{|l|}{ Metastatic focus: } \\
\hline None & $80 \%$ & $70 \%$ & \multirow[t]{3}{*}{$\mathrm{p}=0.441$} \\
\hline Single & $13.3 \%$ & $16.7 \%$ & \\
\hline Multiple & $6.7 \%$ & $13.3 \%$ & \\
\hline \multicolumn{4}{|l|}{ Metastatic site: } \\
\hline None & $80 \%$ & $70 \%$ & \multirow[t]{4}{*}{$\mathrm{p}=0.327$} \\
\hline Bone & $13.3 \%$ & $3.3 \%$ & \\
\hline Others (lung, liver, brain) & - & $2 \%$ & \\
\hline Bone + others & $6.7 \%$ & $6.7 \%$ & \\
\hline \multicolumn{4}{|l|}{ Overall Survival: } \\
\hline Alive & $73.3 \%$ & $56.7 \%$ & \multirow[t]{3}{*}{$\mathrm{p}=0.0001$} \\
\hline Exitus & $13.3 \%$ & $36.7 \%$ & \\
\hline $\mathrm{N} / \mathrm{A}$ & $13.3 \%$ & $6.7 \%$ & \\
\hline
\end{tabular}

of the metachronous cases have the same histopathologic morphology. In bilateral breast cancer cases, metachronous group were mainly diagnosed as invasive ductal or lobular carcinoma. However in synchronous group, other types of breast carcinoma like signet-ring cell differantiated carcinoma, mucinous carcinoma, tubular carcinoma, carcinoma with medullary carcinoma, intracystic papillary carcinoma, apocrine carcinoma were observed especially in one side (Figure 2). The concordance of the histopathologic diagnosis in both breasts were not observed in synchronous. The descriptives and frequencies of the clinical and pathological characteristics of SBBC and MBBC were as shown in Table 2.

When clinical and histopathological parameters were statistically evaluated, ER status, event-free and overall survival were found to be significant between UBC and BBC patients. In comparison of SBBC and MBBC patients, age, histological type of tumor, event-free and overall survival were found to be significant. The mean age of synchronous cases is $59 \pm 8.6$. But in metachronous cases, the mean age is $49.13 \pm 9.78$. The mean age of unilateral cases is $52.79 \pm 12.8$. There was no statistically significance for age when unilateral and bilateral cases compared (independent samples $\mathrm{T}$ test $\mathrm{p}=0.89$ ). But metachronous cases were younger than synchronous cases $(\mathrm{p}=0.005)$. BBC cases had ER expression more than UBC cases $(p=0.043)$. Event-free survival (EFS) is found to be statiscally correlated between unilateral and bilateral cases ( $p=0,009)$ (log-rank test). Event-free survival (EFS) is found to be statically correlated between metachronous 
and synchronous bilateral breast cancer unilateral and bilateral cases $(\mathrm{p}=0,013)$ (log-rank test). SBBC patients had higher overall survival rates than both UBC and $\operatorname{MBBC}(\mathrm{p}=0.0001)$ (Figure 3).

\section{Discussion}

Several studies had reported that $\mathrm{UBC}$ and $\mathrm{BBC}$ types showed various metastatic and prognostic patterns. According to these previous studies, the family history of breast cancer, young age at the time of the first diagnosis, a lobular histology or multicentricity of the first primary breast cancer were found to be increasing the risk of BBC development (Schwenter 2012, Shi 2012). However, metastatic and prognostic patterns of sub-groups of $\mathrm{BBC}$ that are known as SBBC and MBBC have not been well clarified. Therefore, in this study the clinical and pathological parameters were analysed in comparison with UBC and BBC also in subgroups of SBBC and MBBC.

Shi et al, had shown that MBBC patients diagnosed within 24 months after the initial diagnosis had significantly worse prognosis than SBBC patients (Shi, 2012). In this study, the diagnosis age of first metachronous tumor was found to be the earliest in MBBC patients among all other patients. According to the long interval between the first and the second tumor diagnosis in these MBBC patients, we suggest that there should be a different multistep carcinogenetical pathway inducing the occurence of the second tumor. We questioned if the histopathological types of MBBCs differ from each other based on time so first we sub-grouped MBBC as early (time of diagnosis $<36$ months) or late ( $>36$ months). In these subgroups we found that invasive lobular or ductal breast carcinoma types were found to be the most common type among late MBBCs. On the contrary, the late MBBCs patients most commonly had the signet-ring cell carcinoma type. The classification of the signet-ring cell carcinoma type is a conflict whether it is included in ductal or lobular carcinoma. As we found that this complex carcinoma type was more common in late MBBCs even though both breast tissues were exposed to the same carcinogenic effects. During the late interval of the secondary tumor occurence, an additional biological change might be responsible for the transformation to this complex type.

Hartman et al. (2007) showed that SBBC and MBBC diagnosed at different ages showed profound differences in the incidence trends and prognostic outlook (Hartman et al., 2007). In this study, the age of diagnosis did not show statistical significance between UBC and BBC cases however MBBC patients were found to be younger than SBBC patients.

Baykara et al investigated $90 \mathrm{MBBC}$ and $60 \mathrm{SBBC}$ in a retrospective analysis of ten years period (Baykara 2012). They found that MBBC was different from SBBC by having more advanced grade and stage, beside less ER expression, more frequent rates of local relapse and distant metastasis. In their series, MBBC cases responded better to chemotherapy in case of relapse/metastasis. Similarly, we founded that MBBC patients had less ER expression and more frequent rates of single or multipl metastasis than SBBC patients. Also, there was more advanced stage for stage I and II in MBBC. We founded that the most common stage in MBBC was stage II.

Schwentner et al, had studied the association of UBC and $\mathrm{BBC}$ types with prognosis. They showed that the patients with $\mathrm{BBC}$ have primarily a worse prognosis in terms of relaps-free and overall survival than patients with primarily UBC (Schwentner, 2012). Ibrahim et al compared SBBC and MBBC patient characteristics and evaluated their impact on prognosis. They followed up and evaluated 110 cases of BBC between 2005 and 2009. They defined patients as SBBC when contralateral breast carcinoma was diagnosed less than 12 months. According to this evaluation they found that SBBC patients might have worse prognosis (Ibrahim, 2015). Similarly, we evaluated the overall survival of all cases considering the subgroups of UBC, SBBC and MBBC. However, we defined patients with SBBC when contralateral breast carcinoma was diagnosed within 3 months. We found that SBBC patients had higher overall survival rates than both UBC and MBBC. We suggest that the main reason for this finding could be the fact that SBBC patients had underwent bilateral mastectomy.

In this study, we evaluated 768 breast carcinoma cases on the point of view of characteristics of BBCs. $\mathrm{BBC}$ cases were $5.86 \%$ of all cases; $3.91 \%$ was MBBC and $1.95 \%$ was SBBC. Among MBBCs invasive ductal carcinoma subtype was higher than SBBC. Rare types of tumors were observed SBBC cases. BBC cases had ER expression more than UBC cases. The reason of hormonal receptor dependence of $\mathrm{BBC}$ should be explored. These data indicate that $\mathrm{SBBC}$ cases and $\mathrm{MBBC}$ cases might be derived from different carcinogenetic processes. In this series, $\mathrm{BBC}$ cases were found to show worse prognosis than UBC cases. Among BBC, SBBC had the worst prognosis based on overall survival rates. The data about proliferation index, cerbB2 status, stage, nodal status, tumor size or metastasis status were not found to be the reason of this prognostic difference. Our next plan is to compare molecular characteristics of SBBC and MBBC to search for new prognostic

\section{References}

Baykara M, Ozturk SC, Buyukberber S, et al (2012). Clinicopathological Features in Bilateral Breast Cancer. Asian Pac J Cancer Prev, 13, 4571-5.

Chen SF, Du CW, P. Yang, et al (2013). The molecular and clinicopathologic characteristics of bilateral breast cancer. Sci Rep, 3, 2590.

Chen Y, Thompson W, Semenciw R, Mao Y (1999). Epidemiology of contralateral breast cancer. Cancer Epidemiol Biomarkers Prev, 8, 855-61.

Hartman M, Czene K, Reilly M, et al (2007). Incidence and prognosis of synchronous and metachronous bilateral breast cancer. J Clin Oncol, 25, 4210-6.

Holm M, Tjonneland A, Balslev E, Kroman N (2014). Prognosis of synchronous bilateral breast cancer: a review and metaanalysis of observational studies. Breast Cancer Res Treat, 146, 461-75.

Huo D, Melkonian S, Rathouz PJ, Khramtsov A, Olopade OI (2011). Concordance in histological and biological parameters between first and second primary breast cancers. Cancer, 117, 907-15. 
Nuket Eliyatkin et al

Ibrahim NY, Sroor MY, Darwish DO (2015). Impact of bilateral breast cancer on prognosis: synchronous versus metachronous tumors. Asian Pac J Cancer Prev, 16, 100710.

Irvine T, Allen DS, Gillett C, Hamed H, Fentiman IS (2009). Prognosis of synchronous bilateral breast cancer. Br J Surg, 96, 376-80.

Schwentner L, Wolters R, Wischnewsky M, Kreienberg R, Wöckel A (2012). Survival of patients with bilateral versus unilateral breast cancer and impact of guideline adherent adjuvant treatment: a multi-centre cohort study of 5292 patients. Breast, 21, 171.

Senkus E, Szade J, Pieczynska B, et al (2014). Are bilateral breast cancers and breast cancers coexisting with ovarian cancer different from solitary tumors? A pair-matched immunohistochemical analysis aimed at intrinsic tumor phenotype. Pathol Int, 64, 508-17.

Shi YX, Xia Q, Peng RJ, et al (2012). Comparison of clinicopathological characteristics and prognoses between bilateral and unilateral breast cancer. J Cancer Res Clin Oncol, 138, 705.

Yoshimura N, Murakami S, Kaneko M, et al (2013). Synchronous bilateral solid papillary carcinomas of the breast. Case Rep Surg, 2013, 812129. 